\title{
Multi-Velocity Energy-Efficient Reliable Routing Protocol for Coal Mine Monitoring
}

\author{
D.Usha \\ Assistant Professor/CSE Dept \\ National Engineering College \\ K.R.Nagar, Kovilpatti \\ Tamil Nadu, India
}

\author{
B.Paramasivan, Ph.D \\ Professor and Head/CSE Dept \\ National Engineering College \\ K.R.Nagar, Kovilpatti \\ Tamil Nadu, India
}

\begin{abstract}
Wireless sensor network has been used in many real-time applications that require reliable and timely delivery of data This paper presents a real-time communication protocol with data recovery at each hop level to guarantee reliability and also uses the latency of link to determine the shortest path and promises speedy delivery for wireless sensor networks. The proposed protocol provides services such as route discovery based on the reaching speed of the node, multipath forwarding using required reliability and data recovery in single hop. Simulation result shows that the proposed protocol significantly improves the effective capacity of a sensor network in terms of reliability and quickness. Moreover the protocol is highly responsive to the various error conditions experienced and adaptive to large-scale dynamic sensor networks.
\end{abstract}

\section{Keywords}

Wireless Sensor Networks, QoS, multispeed, reliable routing, energy efficiency

\section{INTRODUCTION}

Wireless sensor networks (WSN) are comprised of small devices called sensor nodes with computation, communication and sensing capabilities. It integrates sensor technology, embedded computing, distributed information system and wireless communication technology. Sensor network easily collect sensor data and transmit them to the client, which is called sink node. Users can retrieve information from the sink node to monitor the status of the monitoring region. It has a very broad application in military, business, education, industrial control and environmental control.

Existing coal mine system used wired communication method that makes the system less scalable, because of the long wall and narrow stall system, where it is impossible to wire these sensors [1]. The wired communication requires large amount of wire deployment, which is difficult in poor working conditions. The invention of WSN proposes a new method to settle these problems. In coal mine the concentration of various gases such as methane, wind speed and the underground temperature should be monitored because methane gas explosion is a common cause of coal mine accidents. Thus timely, accurate and reliable gas detection and alert is of great significance.

Under dynamic and heterogeneous environment, the need for adaptability and rapid response to information service systems has become increasingly important. To cope with the continuously changing conditions, timeliness has to be assured by the system. Another necessary requirement is to deliver data reliably [8] due to the lossy nature of wireless transmissions. After deployment it is impossible to change batteries for sensors which are generally battery-powered devices. Hence reduction of energy consumption [12] of nodes also becomes a critical aspect to extend the network lifetime. So the sensors are deployed in the sidewall of the tunnel in such a way that the packet travels reliably and in time to destination only with limited amount of nodes to conserve unwanted energy consumption.

\section{RELATED WORK}

Environment monitoring in underground tunnels has been a crucial task to ensure safe working conditions where many environmental factors, including wind speed, the concentration of various gases, the underground temperature exists[2]. In the coal mine, Electro Magnetic Interference (EMI) is stronger than that above the ground, especially in our country. M.Ding et al [5] proposed a protocol for the rectilinear propagation of high frequent signal where data is transmitted between nodes on the different sides of the wall because the nodes in the same side have worse quality of signal than the nodes in opposite sides. ChiehYih Wan et al [8] proposed a protocol to prioritized the nodes based on the side of the wall so as to minimize the communication between same side nodes. The priority of the opposite side nodes is set to highest for fast recovery of routing. Based on the characteristics of coal mine which is different from traditional network, the reliability and real time of data transfer is very important. Hence the proposed protocol focus on three domains namely reliability, timeliness and energy efficiency.

Ding et al [3] proposed a protocol for knowing the position of the nodes or relative position among them, especially in coal mine to detect, monitor, and check on working attendance.

C.Lu et al[7] proposed a Real-Time Communication Architecture that provides service differentiation in the timeliness domain by velocity-monotonic classification of packets. Based on packet's deadline and destination, its required velocity is calculated and its priority is determined in the velocity-monotonic order so that a high velocity packet can be delivered earlier than a low velocity one.

Speed[13] and MMspeed[15] protocol is designed to provide soft end-to-end deadline guarantees for real-time packets in sensor networks. It ensures a uniformly guaranteed network wide speed to meet end-to-end deadline of packet delivery. However, if workload is too heavy, it is not always possible. In[9] Pagani and Rossi suggested a protocol for reliable broadcast delivery in adhoc networks. Although the protocol is mainly suitable to broadcast are atmost to multicast group, it becomes inadequate for sparse groups. Moreover the protocol requires an underlying clustering protocol, so it is inoperable in nonclustered networks. 
Mobicast [10] aims at reliable and just-in-time delivery of alert packets to all sensor nodes in the moving delivery zone. This service is useful for waking up sensors ahead in the target trajectory being tracked. Di Tian et al [12] proposed a node-scheduling scheme, which can reduce system overall energy consumption, therefore increasing system lifetime, by turning off some redundant nodes. This coverage-based offduty eligibility rule and backoff-based node-scheduling scheme guarantees that the original sensing coverage is maintained after turning off redundant nodes.

F.Ye et al [11], proposed a probing-based density control algorithm to ensure long-lived network where only a subset of nodes are maintained in working mode to ensure desired sensing coverage, and other redundant nodes are allowed to fall asleep most of the time. Working nodes continue working until they run out of their energy or are destroyed. A sleeping node wakes up occasionally to probe its local neighborhood and starts working only if there is no working node within its probing range. Geometry knowledge is used to derive the relationship between probing range and redundancy which is very hard to calculate.

\section{PROPOSED PROTOCOL}

In existing coal mine system, sensor nodes are deployed in deterministic manner where the path is predefined and the location of individual node are already known. Hence only limited paths are there to transmit the observed event data. Due to its harsh environment, nodes are often adapted to failure and an alternate path is chosen for data transfer [3]. But there is only limited choice for path selection. If more number of nodes fails, then the whole network also fails. Hence one should manually redeploy the nodes in the failure area for the network to operate again. This is very difficult due to harsh conditions in coal mines.

In the proposed method the protocol uses the nondeterministic method where large numbers of nodes are randomly deployed on the two walls of the coal faces and the paths are not known well in advance. Each node identifies its location and determines a path to its immediate neighbor whenever there is a need to transmit the data. If node failure occurs an alternate node is chosen and there are many choices for the data to reach the destination. Since monitoring in underground tunnels has been a crucial task, any drastic change should be immediately informed for necessary action. To prolong the network lifetime with nodes having little battery power some measure for energy conservation is also essential. Therefore any data observed should reach within short time in a reliable manner. The proposed protocol focuses on three domains namely timeliness, reliability and energy efficiency.

Whenever an event like methane explosion or fire is detected it immediately identifies a neighbor to communicate. Considering the rectilinear propagation of high frequent signal, the nodes in the opposite side of the wall are chosen for data transfer because the quality of the signal will be worse if the data are transferred to the same side of the wall. Hence to identify the side of the wall each node maintains a value $s$ (side) and p(priority) in Basic Information Table (BIT) to ensure the quality of communication. If the s value of one node is 0 then the $s$ value of another node deployed in different side of wall is 1 . Hence every time a node is chosen for data transfer, the information in the BIT table is referred.

The priority $\mathrm{p}$ is based on some conditions. The nodes at the other side which is close to the requesting node have higher priority than far ones. Hence farther nodes at the opposite side are given second priority. But the nodes in the same side are given lowest priority. The data in the BIT is very important in high reliable routing protocol for route recovery and data transmission. If no node is found in the forwarding direction, a warning message is triggered back to the immediate sender to reroute the packet. The sender then re-chooses other neighboring nodes to identify a possible route around the communication hole [14].

\subsection{Realization in timeliness realm}

Considering the timeliness domain the data must reach the destination within the end-to-end deadline. The end-to-end deadline for a packet is the maximum time within which the packet should reach the destination. Different sensory data has different deadlines depending on the dynamics of the sensed environment. For eg. The event of fire has shorter deadline than the gas leakage. Here local decisions are made at each hop level to meet out the required deadline. For this the source node $\mathrm{S}$ selects a node with the most proper speed [8] for a packet $\mathrm{x}$ to reach its destination based on the distance to the destination D. To meet the end-to-end deadline the minimum required speed level for a packet $\mathrm{x}$ is calculated as

Req.Speed $=\operatorname{dist}(\mathrm{S}, \mathrm{D}) / \operatorname{deadline}(\mathrm{x})$

\section{where dist $(\mathrm{S}, \mathrm{D})$ - Distance of a packet to destination deadline $(\mathrm{x})$ - end-to-end deadline of a packet}

The packet is forwarded to a high priority node chosen from the opposite whose progress speed is higher than the required speed and also whose distance from the sender is half of the transmission range of a node [13]. The node which is too far may tempt to loose some data. The node with highest Progress Speed and not too far is selected to achieve timeliness. The Progress Speed is calculated using the formulae,

Prog.Speed=dist(S,D)-dist(I,D)/ delay(S,I)

If the nodes cannot find any node with greater Progress Speed than the ReqSpeed, the nodes issue "back-pressure packets" to reduce the incoming packets traffic from other neighboring nodes.

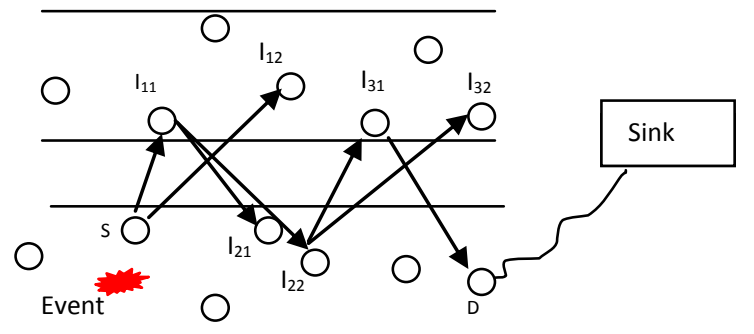

Fig.1: Virtual overlay of multiple speed layers

Consider the Fig.1, an event is detected by the source node $\mathrm{S}$ and it calculates the Progress Speed for the next two neighboring nodes I11 and I12. The Progress Speed of I11 and I12 are higher than that of the ReqSpeed, so both are eligible for forwarding the packet. But I11 is selected as next node because the Progress Speed is greater than that of I12. This in turn selects the node I22 in the opposite direction and reaches the destination through $\mathrm{I} 31$.

\subsection{Updating of Required Speed}

Suppose the intermediate nodes, due to congestion, face a longer delay and if the deadline is less, then a new required speed is calculated. This is done by comparing the expected 
latency and the remaining time to deadline. If the time needed to reach the destination is larger than the remaining time, then the required speed should be booted up to achieve the deadline. To handle this problem the elapsed time is calculated whenever the node receives the last bit of the packet and also when the node transmit the first bit to physical link. Using this the remaining time to deadline is also calculated. The elapsed time is calculated by using the following formula.

$t^{\text {elapsed }}=t^{\text {departure }}+t^{\text {transdelay }}-t^{\text {arrival }}$.

$\mathrm{t}^{\text {departure }}$ - time when the node transmits the first bit of a packet to the physical link.

$\mathrm{t}^{\text {transDelay }}-$ transmission delay of the packets

$\mathrm{t}^{\text {arrival }}$ - the time when the node receives the last bit of the packet

Hence the remaining time to dead line is calculated using the elapsed time by the following formula

$\operatorname{Re}$ m.time $(x)=$ deadline $(x)-t^{\text {elapsed }}-t^{\text {propdelay }}$. two nodes.

Where $\mathrm{t}^{\text {propDelay }}$ - the propagation delay between

If any node notices that the current speed is insufficient to meet the remaining time to deadline then the speed level is boosted by the following formula

$$
\operatorname{Re} \text { q.Speed }(x)=\frac{\underset{m, d}{\operatorname{dis}}(x)}{\operatorname{Re} m \cdot \operatorname{deadline}(x)}
$$

Where dist $t_{\mathrm{m}, \mathrm{d}}(\mathrm{x})$ - distance between the intermediate node and the final destination node.

\subsection{Multipath forwarding by dynamic compensation}

Although timeliness plays an important role in coal mining system, reliable routing also plays an equal role because nodes are very easily damaged even in minor disaster. So whenever there is node failure, packet drops and errors on wireless links are very common. So an alternative path is preferable for guaranteed delivery of data to the destination [15]. This is also used for load balancing and to avoid hot spots on shortest path. Here the protocol issues multipath forwarding technique using dynamic compensation at each local hop level.

To achieve reliable transport each node maintains an average reaching probability by considering the packet loss (including packet drops and error on the wireless links) percentage at each path. The local reaching probability of a packet is calculated using

$\operatorname{RP}^{\mathrm{d}} \mathrm{ij}=\left(1-\mathrm{e}_{\mathrm{ij}}\right)\left(1-\mathrm{e}_{\mathrm{ij}}\right)^{[\mathrm{dist} \mathrm{j}, \mathrm{d} / \mathrm{distij}]}$

Where $\left[\right.$ dist $\left._{\mathrm{j}, \mathrm{d}} / \mathrm{dist}_{\mathrm{ij}}\right]$ indicate hop count estimation from node $\mathrm{j}$ to final destination.

When the reaching probability is less than the required probability, the node adopts more than one path to forward data. Then the total reaching probability (TRP) of the packet that will successfully deliver it to the destination is calculated by
$\mathrm{TRP}=1-(1-\mathrm{TRP})\left(1-\mathrm{RP}_{\mathrm{ij}}^{\mathrm{d}}\right)$

The two neighbor nodes whose combined TRP is larger than the required reachability $\left(\mathrm{p}^{\text {req }}\right)$ are included for packet forwarding. The dynamic compensation is used at each hop level to find out the multiple forwarding nodes.

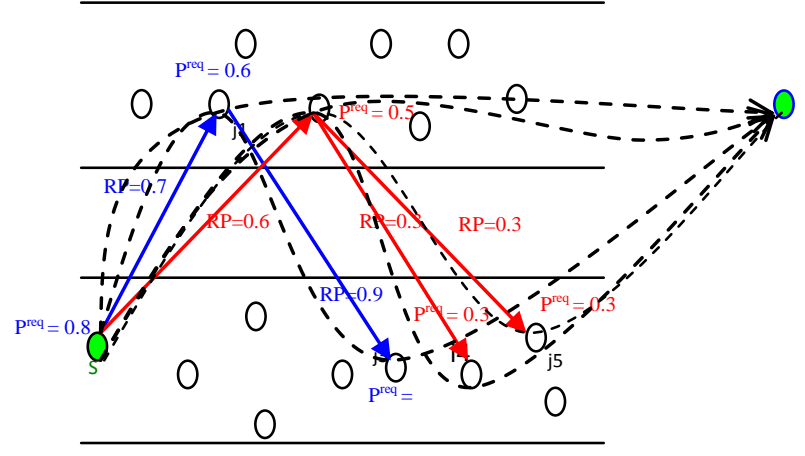

Fig .2. Multipath Forwarding and Dynamic Compensation

Here a sensor node $\mathrm{S}$ detects an event with reachability preq $=80 \%$, it forwards the packet to two immediate neighbours $\mathrm{j} 1$ and $\mathrm{j} 2$ based on its local reachability RPds $\mathrm{j} 1=70 \%$ and RPds $\mathrm{j} 2=60 \%$. Hence the total reaching probability $\mathrm{TRP}=0.88$, which is higher than reachability requirement. Therefore the packets are transformed to the two nodes $\mathrm{j} 1$ and $\mathrm{j} 2$ with reachability requirement preq $=0.6$ and preq $=0.5$ respectively, so that the TRP is 0.8.Again these nodes $\mathrm{j} 1$ and $\mathrm{j} 2$ make local forwarding decisions such that $\mathrm{j} 1$ forwards to $\mathrm{j} 3$ with local reachability $\operatorname{RPdj} 1 \mathrm{j} 3=0.9$ which is higher than the required reachability, whereas $j 2$ forwards to $\mathrm{j} 4$ and j5 with local reachability $\mathrm{RPdj} 2, \mathrm{j} 4=0.3$ and $\operatorname{RPdj} 2, \mathrm{j} 5=0.3$ which is less than the required reachability 0.6 . Hence forwarded to both $\mathrm{j} 4$ and $\mathrm{j} 5$ and $\mathrm{TRP}=0.51$ is now satisfied.

In this way the node dynamically compensate the previous wrong decision as the packet travels to the final destination. If the receiving node cannot find a neighbor node to satisfy the reachability, it employs a back pressure mechanism to reduce reliability expectation of previous node.

\subsection{Discrimination in Energy Efficiency}

Other than timeliness and reliability, energy efficiency also plays an important role in coal mining system. Experimental measurements have shown that generally data transmission is very expensive in terms of energy consumption [5], while data processing consumes significantly less.

Our protocols take the most intuitive approach to conserve unwanted power depletion. The basic idea is that a node should wakeup [6] only when another node wants to communicate with it. The main problem here is to inform the sleeping node to communicate with it. For this problem the protocol use mulutiple radios with different energy and performance tradeoffs (i.e. a low-rate and low-power radio for signaling, and a high rate but more power hungry radio for data communication). The implementation of such schemes typically requires two different channels: a data channel for normal data communication, and a wakeup channel for awaking nodes when needed. Other nodes are in sleeping mode until it receives a packet from a neighboring node. In such scenarios sensor nodes are in the monitoring state (i.e., they only sense the environment) for most of the time. As the 
nodes remain active only for a minimum time period, energy consumption is considerably reduced.

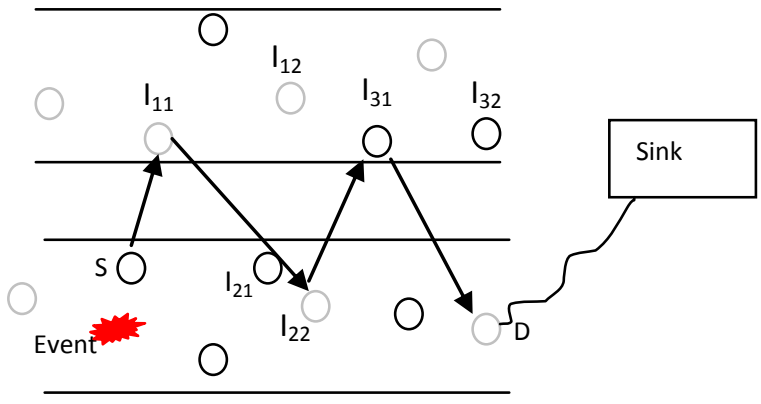

Fig.3. Illustration of Sensor states

Consider the scenario, the source node wants to communicate with the node I11 in the opposite direction which is sleeping. In this occasion the radio signals are passed through the wakeup channel by the transceiver to make it active. The sleeping nodes are represented as light circles and the active nodes are represented as dark circles.

\section{SIMULATION AND RESULTS}

This section presents the performance results of the designed MERR protocol compared with various other existing protocols such as Speed [8] and PSFQ [7] obtained using NS2 simulation. The results are measured in terms of network lifetime, reachability ratio and delivery ratio for a fixed size of network. To evaluate the performance of the MERR protocol in a realistic scenario, the sensor nodes are deployed randomly. Nodes use radios with 2 mbps bandwidth with a nominal radio range of $20 \mathrm{~m}$. The channel access is the simple Carrier Senses Multiple Access/Collision Avoidance (CSMA/CA), and a uniformly distributed channel error model is used. A user node attempts to inject a program image file of a size equal to $2.5 \mathrm{~kb}$ to another node. The packet size is 512 bytes. Packets are generated from the user node and transmitted at the rate of one packet every $50 \mathrm{~ms}$.

Fig.4. shows the delivery ratio in respect to time interval. The delivery ratio of the proposed protocol MERR is found to be elevated than some of the standard protocols such as Speed and PSFQ. For a period of $100 \mathrm{sec}$ there is a maximum delivery ratio of $96 \%$ for the EMR whereas the ratio is 90 and 93 for PSFQ and Speed.

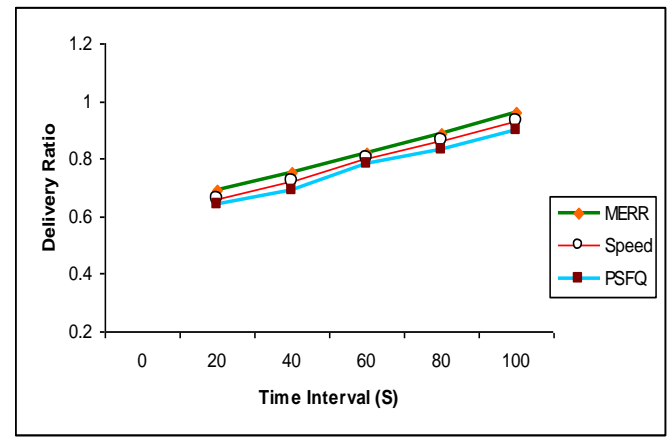

Fig.4. Delivery Ratio of Packets

Fig.5. shows the network lifetime as a function of the number of rounds. It is observed that as the number of rounds increases, the lifetime of the network decreases. From the simulation it is apparent that, the lifetime of the nodes are extended in the proposed protocols when compared with other two protocols.

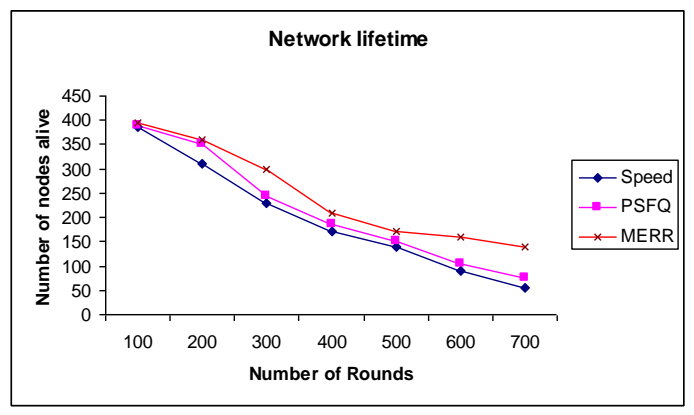

Fig.5. Network Lifetime

\section{CONCLUSION}

Based on the characteristics of coal mine, a new routing protocol is designed which makes the network more suitable for the surroundings of coal mine. This protocol proposes a novel packet delivery mechanism called MERR for wireless sensor networks to provide service differentiation and probabilistic QoS guarantees in the timeliness, energy efficient and reliability domains. For the timeliness domain, the protocol provide multiple network-wide speed options so that various traffic can dynamically choose the proper speed options for their packets depending on their end-to-end deadlines. To conserve energy and to increase system lifetime, some redundant nodes are turned off using off-duty eligibility that guarantees the original sensing coverage to maximum possible extent. For the reliability domain, the proposed protocol use proactive multipath forwarding to control the number of packet delivery paths depending on the required end-to-end reaching probability.

\section{REFERENCES}

[1] X.D.Wang, X.G.Zhao, Z.Liang,et al, "Deploying a Wireless Sensor Network on the Coal Mines". In Proceeding of the IEEE International Conference on Networking, Sensing and Control, 2007.

[2] B.Meng, Z.xiaoguang, Z.G.Hou and T.Min, "A Wireless Sensor Network Used in Coal Mines" in proceedings of IEEE Networking, Sensing and Control, London, UK, April. 2007.

[3] E.J.Ding, M.Y.Qing, P.Y.Gao, Q.Zhou, "Improved Pattern Matching Localization of WSN in Coal Mine" in proceeding of the IEEE International conference on Information Acquisition July.2007, Jeju City, Korea.

[4] Y.L.Chih, C.W.Peng and C.T.Yu, "Efficient in network moving object tracking in wireless sensor networks" in Proceedings of IEEE Trans. Mobile Computing Aug.2006.

[5] M.Ding, D.Chen, K.Xing and X.Cheng, "localized FaultTolerant Event Boundary Detection in Sensor Network" in Proceedings of IEEE INFOCOM, 2005

[6] C.Y.wan, S.B.Eisenman, A.T.Campbell and J.Crowcroft, "Siphon: overload traffic management using multi-radio virtual sinks in sensor networks" in Proceedings of ACM SenSys, 2005.

[7] C. Lu, B.M. Blum, T.F. Abdelzaher, J.A. Stankovic, and T. He, "RAP: A Real-Time Communication Architecture for Large-Scale Wireless Sensor Networks," Proc. IEEE 
Real-Time and Embedded Technology and Applications Symp. (RTAS 2002), Sept. 2002.

[8] Chieh-Yih Wan, Andrew T. Campbell, and Lakshman Krishnamurthy, "PSFQ: A Reliable Transport Protocol for Wireless Sensor Networks", September 28, 2002, Atlanta, Georgia, USA. Copyright 2002 ACM 1-58113589-0/02/0009.

[9] E.Pagani and G.P.Rossi ,"Reliable Broadcast in Mobile Multihop Packet Networks" Proceddings of ACM/IEEE, MOBICOM97, Budapset, Hungary, Sept.1997, pp34-42

[10] Q. Huang, C. Lu, and G.-C. Roman, "Spatiotemporal Multicast in Sensor Netwroks," Proc. First Int'l Conf. Embedded Networked Sensor Systems, Nov. 2003.

[11] F. Ye, G. Zhong, J. Cheng, S. Lu, and L. Zhang. Peas: A robust energy conserving protocol for long-lived sensor networks. In Proceedings of the 23rd International Conference on Distributed Computing Systems (ICDCS '03), pages 28-37, 2003.
[12] Di Tian, Nicolas D. Georganas Energy efficient routing with guaranteed delivery in wireless sensor networks. Proceddings of IEEE.2003 pp1923-1929

[13] T. He, J. Stankovic, C. Lu, and T. Abdelzaher, "SPEED: A Stateless Protocol for Real-Time Communication in Sensor Networks," Proc. IEEE Int'l Conf. Distributed Computing Systems, pp. 46-55, 2003.

[14] Dong Jiang,Qianping Wang, Yan Zhao,Ke Wang, "The Research and Design of High Reliability Routing Protocol of Wireless Sensor Network in Coal Mine", 2009 International Conference on Networks Security, Wireless Communications and Trusted Computing.

[15] Emad Felemban, Chang-Gun Lee, and Eylem Ekici, "MMSPEED: Multipath Multi-SPEED Protocol for QoS Guarantee of Reliability and Timeliness in Wireless Sensor Networks ," Proc. IEEE Transactions on mobile computing, Vol.5, No. 6, June 2006. 